\title{
SPD und Gewerkschaften: Vom Wandel einer privilegierten Partnerschaft
}

\author{
Wolfgang Schroeder
}

Das über Jahrzehnte hinweg lose, aber beständige Bündnis zwischen SPD und Gewerkschaften war für die politischen Kräfteverhältnisse und die politisch-kulturellen Allianzen in der Bundesrepublik stilbildend - trotz immer auch vorhandener Konflikte zwischen den beiden ungleichen Partnern. Sind also die Spannungen, die es seit einigen Jahren im Verhältnis von SPD und Gewerkschaften ganz offensichtlich gibt, nichts anderes als eine vorübergehende Beziehungskrise? Oder zeigen sich hier Symptome eines unumkehrbaren Entfremdungsprozesses? Und was wären die Folgen für die SPD, die Gewerkschaften wie auch für das politische System in Deutschland?

\section{Einleitung}

Auffallend an den jüngeren Kontroversen seit der „Agenda 2010“ ist, dass sowohl Gewerkschaften als auch Sozialdemokratie aus einer Position der Schwäche agieren. Beide haben ihre eigenen, jedoch nicht unähnlichen Bestands- und Akzeptanzprobleme - und werfen sie sich zugleich gegenseitig vor. Auch wenn es die SPD 2005 geschafft hat, wieder an der Regierung beteiligt zu sein, sind ihre Nöte offensichtlich: hohe Mitglieder- und Wählerverluste, gerade im Milieu gewerkschaftlich organisierter Arbeitnehmer, und eine trotz Programmdebatte und neuem Grundsatzprogramm nicht abgeschlossene Identitätssuche, die sich nach der Etablierung eines Fünfparteiensystems auf Länderebene sogar noch verschärft hat. Aber auch die Gewerkschaften haben an Stärke eingebüßt. Sie sind politisch in der Defensive. In der Tarifpolitik versuchen sie, unter schwierigen Rahmenbedingungen die Verteilungsposition der Arbeitnehmer zu sichern und Arbeitsplätze zu halten. Hinzu kommen Mitgliederrückgänge, die Alterung der Mitgliedschaft und eine bis heute nicht gelungene Adaption des wirtschaftlichen Strukturwandels hin zu einer modernen Wissens- und Dienstleistungsökonomie innerhalb der gewerkschaftlichen Mitgliedschaft.

Im wissenschaftlichen und politischen Diskurs sind namhafte Stimmen zu hören, die die Distanzierung der Sozialdemokratie (in ganz Europa) von den Gewerkschaften als eine Bedingung für die erfolgreiche Transformation der Sozialdemokratie sehen. Die sozialdemokratische Formel „mehr
Markt - weniger Staat" und die strategische Flexibilität sozialdemokratischer Parteien auf dem Weg zur Regierungsübernahme bzw. in Regierungspositionen lieBen sich nur durch die Emanzipation von den Ansprüchen und Interessen der Gewerkschaften durchsetzen. Dieser These von einer zunehmenden (und zunehmend notwendigen) Distanzierung steht die Einschätzung gegenüber, dass es im Verhältnis zwischen Gewerkschaften und Sozialdemokratie immer schon wechselhafte Phasen gab: ein Spannungsbogen, der von größerer Übereinstimmung bis hin zu angespannten, ja konfliktreichen Beziehungsmustern reicht. Deshalb könne man keinesfalls von einer linearen Erosionsentwicklung sprechen (Armingeon 1988, S. 130). Einmütigkeit habe sich - so Schneider (1994) - ohnehin nie gezeigt, und eine inhaltliche oder strategische Alternative zur Zusammenarbeit sei - unter der Perspektive machtpolitischer Durchsetzungsfähigkeit - letztlich nicht in Sicht.

James Piazza (2001) kommt hinsichtlich des Verhältnisses zwischen Gewerkschaften und sozialdemokratischen Parteien in einer internationalen Vergleichsstudie zu dem Ergebnis, dass vor allem die Globalisierung die Sozialdemokratie zu einem eher sozialliberalen Politikstil gezwungen habe. Die klassische Beziehung von Gewerkschaften und sozialdemokratischen Parteien werde dadurch komplett „entbunden“ (de-linked). Stephen S. Silvia (1992) geht demgegenüber schlichtweg von einer Abnutzung des Verhältnisses aus, die durch die neue de-ideologisierte und rational-karriereorientierte Generation in der SPD vorangetrieben werde.

So sind Gewerkschaftsskeptiker in der SPD fest davon überzeugt, dass schon die unterstellte Nähe der Gewerkschaften zur
SPD schädlich für deren Mehrheitsfähigkeit sei. Denn das gesellschaftliche Image der Gewerkschaften sei so schlecht und die Mobilisierungsfähigkeiten der Gewerkschaften seien so schwach, dass die SPD von deren Unterstützung sowieso keinen Nutzen habe. Während diese beiden Gruppen die Kosten eines Endes der konstruktiven Beziehungen zwischen SPD und Gewerkschaften für gering erachten, rechnet die Mehrheit mit einer modifizierten Kontinuität, die beiden weiterhin Vorteile verschaffen könnte.

In diesem Beitrag wird die These vertreten, dass die seit einigen Jahren offensichtlichen Spannungen zwischen SPD und Gewerkschaften nicht nur den Konjunkturen der jeweiligen politischen Prioritäten und programmatischen Ziele geschuldet sind, wenngleich auch dies eine wichtige Rolle spielt. Vielmehr gibt es eine tiefer reichende Kluft, die sich aus den sozialstrukturellen Verschiebungen zwischen der gesellschaftlichen, der gewerkschaftlichen sowie der sozialdemokratischen Mitgliederstruktur ergibt. Während sich die SPD von ihrer Arbeiterherkunft gelöst hat und der Anteil der anderen sozialen Gruppen nahe$\mathrm{zu}$ ständig gestiegen ist, blieb bei den Gewerkschaften ein ähnliches Wachstum von sozialen Milieus und Gruppen jenseits der gewerblichen Arbeiterschaft bislang aus. Doch anders als in den vorgenannten Ein-

Wolfgang Schroeder, Prof. Dr., Universität Kassel, Lehrstuhl "Politisches System der Bundesrepublik Deutschland/Staatlichkeit im Wandel". Arbeitsschwerpunkte: Arbeitsbeziehungen, Verbände, Parteien und Sozialstaatspolitik.

e-mail:wolfgang.schroeder@uni-kassel.de 
schätzungen werden die Folgen dieser Drift kritisch gesehen: Nach Lage der Dinge kann sich der definitive Bruch einer lose verkoppelten Bezugnahme zwischen Gewerkschaften und Sozialdemokratie für beide Seiten negativ auswirken: Für die Sozialdemokratie hinsichtlich ihrer Mobilisierungs- und Mehrheitsfähigkeit; für die Gewerkschaften hinsichtlich einer realistischen Gestaltungspolitik, die in vielen Bereichen einer staatlich flankierten Ressourcenpolitik bedarf.

\section{Phasen und Probleme einer Beziehung}

Zeichnet man die historischen Entwicklungslinien des SPD-Gewerkschaftsverhältnisses nach, so lassen sich für Deutschland fünf Phasen unterscheiden, wobei die wesentlichen Unterschiede sich daraus ergeben, ob die SPD an der Regierung beteiligt ist und ob es um einen Zuwachs oder einen Abbau sozialstaatlicher Leistungen geht.

Phase 1 beschreibt die grundsätzliche Neuordnung und Klärung der Beziehungen zwischen Gewerkschaften und Parteien, die von der Gründung parteipolitisch unabhängiger Einheitsgewerkschaften 1945 bis Mitte der 1960er Jahre reichte. In dieser Zeit war die SPD in der Opposition, und beide Organisationen erlebten ihre ersten grundlegenden programmatischen und organisatorischen Transformationsprozesse.

In der zweiten Phase, die zwischen 1966 und 1974 verortet werden kann, machte die SPD ihre ersten Erfahrungen als Regierungspartei. Die Gewerkschaften wurden als politischer Mitgestalter gewonnen (z. B. in der Konzertierten Aktion 1967-1977), was für beide Akteure in Zeiten sozialstaatlicher Expansion Nutzen stiftete (z. B. die Rechte und Stellung der Gewerkschaften im Betriebsverfassungsgesetz 1972; ihre Unterstützung der SPD-Politik bei den Ostverträgen) und keine existenziellen Belastungsproben mit sich brachte.

In der anschließenden dritten Phase fand man sich in einer „Kooperationskrise“ wieder. Die Politik sah sich durch das Ende des Wirtschaftsbooms neuen Herausforderungen gegenüber, die sie unter anderem durch eine stärkere Einbindung der Gewerkschaften zu lösen versuchte. Nachdem dies scheiterte und die Spannungen zunahmen, kam es Anfang der 1980er Jahre sogar zu ersten Demonstrationen der Gewerkschaften gegen die Sparpolitik einer SPDgeführten Regierung.

Die vierte Phase, die durch die Oppositionsrolle der SPD zwischen 1982 und 1998 gekennzeichnet war, führte einerseits zu einer neuen Annäherung, was sich auch deutlich im Berliner Programm von 1989 niederschlug; andererseits gelang es nicht, neue inhaltliche, strategische Verabredungen zu treffen. In der Zeit des deutschen Einigungsprozesses kam es zu deutlichen Spannungen, weil die Gewerkschaften die Einigungspolitik der Kohl-Regierung durch eigene organisationspolitische Aktivitäten unterstützten, während große Teile der Sozialdemokratie noch Skepsis und Vorbehalte gegenüber der schnellen Einheit hegten. Im Laufe der 1990er Jahre verbesserten sich die Beziehungen wieder.

Die fünfte Phase umfasst die Zeit sozialdemokratischer Regierungsbeteiligung (seit 1998), wobei infolge des Umbaus des Wohlfahrtsstaates und divergierender Prioritäten nahezu dauerhaft Spannungen bestanden, die weder durch das „Bündnis für Arbeit" (1998-2003) noch durch ein neues, deutlich verbessertes Betriebsverfassungsgesetz (2001) nachhaltig abgebaut werden konnten. Im Gegenteil: Latente Konflikte führten ab 2003 infolge der Agenda 2010 zu einem starken Entfremdungsprozess, dessen Folgen eine kleine Gruppe von Gewerkschaftsfunktionären dazu nutzte, eine links-traditionalistische Alternative zur SPD zu gründen. Hiermit wollten sie gewissermaßen die Lücke, die aus ihrer Sicht „new labour" aufgerissen hatte, durch eine neue „old labour"-Alternative besetzen. Mit der großen Koalition hat sich das Verhältnis zwar wieder entspannt, was allerdings bislang weder zu neuer Selbstsicherheit noch zu neuen Kooperationsperspektiven geführt hat.

Auch wenn die tiefer liegenden Probleme in der Beziehung zwischen SPD und Gewerkschaften primär durch sozialstrukturell geprägte Interessen und Politikoptionen geprägt sind und somit eine objektive Kluft darstellen, so kann als verbleibendes Bindeglied die Bedeutung wechselseitiger Kommunikation nicht hoch genug bewertet werden. Zugespitzt könnte man formulieren: Ein intensiver Austausch kann einen Teil der Diskrepanz heilen. Dabei kommt den jeweiligen Eliten der Parteiund Gewerkschaftspolitik große Bedeu- tung zu. Denn es gibt vielfältige inhaltliche und personelle Verbindungslinien zwischen Gewerkschaften und Sozialdemokratie, sodass sich empirisch die These von einem bevorstehenden Bruch zwischen beiden nicht begründen lässt. Das bedeutet im Umkehrschluss allerdings nicht, vorhandene Probleme verharmlosen zu können. Im Folgenden werden die nach wie vor bestehenden Verbindungslinien zwischen SPD und Gewerkschaften wie auch vorhandene Risse genauer betrachtet, um anschließend auszutarieren, was die neue Konstellation von zwei Parteien mit sozialdemokratischen Wurzeln im deutschen Bundestag für die Zukunftsperspektiven von SPD und Gewerkschaften sowie ihr Verhältnis zueinander bedeuten kann.

\section{Die Ist-Situation}

\subsection{DIE DGB-GEWERKSCHAFTEN}

Die deutschen Gewerkschaften erlebten in der sogenannten Phase der fordistischen Massenproduktion, also etwa zwischen 1955 und 1985, ihr "goldenes Zeitalter" als selbstbewusster und öffentlich anerkannter Akteur. Seither versuchen sie sich organisatorisch und konzeptionell auf die Herausforderungen des postindustriellen Kapitalismus einzustellen. Sie agieren politisch in drei Dimensionen: Erstens sind sie in das politische System partiell eingebunden (Konsultations- und Einflussmöglichkeiten) und besitzen zugleich besondere $\mathrm{Zu}$ gangsgarantien durch das Mitbestimmungs- und Betriebsverfassungsrecht sowie ihre besondere Stellung bei den Sozialversicherungen. Zweitens agieren sie als Interessengruppen, die die Anliegen der Beschäftigten gegenüber der Regierung und den Parteien vertreten. Drittens handeln sie bisweilen partiell auch wie eine soziale Bewegung, wenn sie sich mit anderen Gruppen für gesamtgesellschaftliche Ziele engagieren.

Unabhängig davon, ob die Gewerkschaften als korporatistischer Akteur, als Interessengruppe oder als soziale Bewegung auftreten, hängt ihr Einfluss in diesen jeweiligen Arenen und Rollen davon ab, ob sie eine hohe Mitgliederzahl hinter sich bzw. ein nachhaltiges Vetopotenzial haben. Die hohe und repräsentative Mitgliederzahl ist wichtig, um die finanziellen Res- 
sourcen - vom Personal bis hin zu den Streikkassen - zu sichern. Darüber hinaus sind sie neben einer passiven Folgebereitschaft der Mehrheit ihrer Mitglieder auf die aktive Mitarbeit eines relevanten Mitgliederanteils angewiesen. Das sie auf Letzteres nicht mehr selbstverständlich vertrauen können, zeigt sich unter anderem in der Krise des Ehrenamtes. Die Gegenwartsdiagnose ist eindeutig: Ein drastischer Mitgliederrückgang gefährdet die politische Handlungsfähigkeit der Gewerkschaften. Nachdem in den 1950er Jahren noch vom „Wunder der Organisation“ (Pirker 1960) gesprochen wurde und ein Nettoorganisationsgrad (nur erwerbstätige Mitglieder) von $36 \%$ und ein Bruttoorganisationsgrad von etwa $40 \%$ erreicht wurde, stand bereits in den 1960er Jahren die Frage auf der Tagesordnung, wie es den Gewerkschaften gelingen könnte, ihre Mitgliederstruktur mit dem wirtschaftlich-gesellschaftlichen Wandel zu synchronisieren (Müller-Jentsch/ Ittermann 2000, S. 91). In den 1970er Jahren gelang es den Gewerkschaften zwar trotz einer bereits damals einsetzenden Verschiebung zwischen und innerhalb der Branchen, das Organisationsniveau auszudehnen, indem sie das gewerbliche Arbeiterpotenzial verstärkt integrierten. $\mathrm{Zu}$ gleich wurden Nicht-Erwerbstätige wie Rentner und Arbeitslose als Mitglieder aufgenommen. Deren Anteil in den DGB-Gewerkschaften liegt heute bei knapp über $30 \%$. Angesichts eines sich ändernden Arbeitsmarktes war diese Mitgliederpolitik defensiv, denn die Anpassung an die veränderte Arbeitsmarktdynamik wurde damit verfehlt. Während sich die Beschäftigung im produzierenden Gewerbe drastisch reduzierte (von ca. 56 \% im Jahr 1965 auf etwa $25,4 \%$ im Jahr 2007), hat sie sich im Dienstleistungsbereich mehr als verdoppelt und ist auf ca. 72,4 \% angestiegen. Zwar ist auch in den DGB-Gewerkschaften der Angestelltenanteil gewachsen, gleichwohl verlief dieses Wachstum nicht synchron zum realen Anstieg der Angestellten unter den Beschäftigten. Die gewerkschaftliche Mitgliederstruktur bildet also nicht mehr die Sozialstruktur des Arbeitsmarktes und der Gesellschaft ab. Angesichts des gewerkschaftlichen Selbstverständnisses, mitten in der Gesellschaft die Interessen der Mehrheit aller Beschäftigten zu vertreten, wird dieser Sachverhalt zu einem zentralen Problem.

\subsection{DIE SPD}

\section{VERÄNDERUNGEN IM WÄHLERPOTENZIAL}

Mit dem Godesberger Programm von 1959 wandelte sich die SPD auch programmatisch von einer Klassen- zu einer Volkspartei. Damit trug sie nicht nur dem sozialstrukturellen Wandel auf dem Wählermarkt Rechnung. Schaut man sich die Daten zu Gruppenbindung und Wahlverhalten an, so lassen sich daraus vor allem zwei Ergebnisse festhalten: Erstens, die sozialstrukturelle Basis der Sozialdemokratie bilden nach wie vor die Arbeitnehmerwähler; zweitens, wählen von ihnen gruppenbezogen betrachtet die gewerkschaftlich organisierten Arbeiter am häufigsten SPD (Wessels 2000).

Während das SPD-Wahlergebnis bei der Bundestagswahl 2005 unter dem Arbeiterwähleranteil lag, entschieden sich Gewerkschaftsmitglieder zu 12,8 \% und gewerkschaftlich organisierte Arbeiter sogar zu 17,9\% öfter an den Wahlurnen für die SPD. Ein klarer Trend, der nach oben oder unten zeigt, lässt sich aus diesen Daten im zeitlichen Verlauf allerdings nicht erkennen. Etwas anders sieht es bei der Frage hinsichtlich der Neigung zur SPD aus, also der Parteisympathie (ARD/Infratest dimap Wahlbefragung 2005).

Gerade bei den treuesten Stammwählern ist hinsichtlich der Parteisympathie ein signifikanter Rückgang festzustellen: In einem Zeitraum von 15 Jahren hat sich die Neigung gewerkschaftlich organisierter Arbeiter zur SPD halbiert. Besonders brisant fielen die Wahlverluste der SPD bei der Bundestagswahl 2002 im Vergleich zur 1998er Wahl aus. Dieser Trend setzte sich bei der Bundestagswahl 2005 fort, bei der die SPD unter den Arbeitern um 5 Prozentpunkte auf $37 \%$ abrutschte, aber auch die CDU verlor 7 Prozentpunkte $(30 \%)$. Gewinner war die Linkspartei, die sich um 7 Prozentpunkte auf $12 \%$ verbesserte. Bei den gewerkschaftlich organisierten Arbeitern sah es ähnlich aus: Während SPD (von $54 \%$ auf $51 \%$ ) und CDU (von $26 \%$ auf $21 \%$ ) deutliche Einbußen hinnehmen mussten, stieg der Anteil der gewerkschaftlich organisierten Arbeiterwähler bei der Linkspartei (von $4 \%$ auf $14 \%$ ) deutlich an (ebd.).

Der Akzeptanzverlust bei den Arbeitern kann nicht nur unter quantitativen Gesichtspunkten diskutiert werden. Denn wenn es nicht mehr gelingt, diese Gruppe $\mathrm{zu}$ integrieren, so hat dies auch erhebliche Konsequenzen für das Profil und Image der SPD im Parteienwettbewerb. Diese These gewinnt mit der Existenz einer zweiten sozialdemokratischen Partei im Parlament, die in weit stärkerem Maße im Bereich der Umverteilungs- und Sozialstaatspolitik egalitäre Positionen vertritt, noch größere Bedeutung. Zugleich ist aber auch offensichtlich, dass in einer derart differenzierten, sozial und kulturell gespaltenen Arbeitnehmergesellschaft eine schlichte Re-Orientierung auf das klassische Arbeiterpotenzial für die Mehrheitsfähigkeit der SPD falsch wäre, zumal dann, wenn sich daraus eine nachhaltige Schwächung in anderen Wählersegmenten ergeben würde. Tatsächlich haben in den letzten Jahrzehnten bedeutsame Verschiebungen zwischen den Wählersegmenten stattgefunden, die auch von den Gewerkschaften nicht ignoriert werden können. Das Reservoir aus unorganisierten Arbeitern und Gewerkschaftsmitgliedern (Arbeiter wie Nicht-Arbeiter), aus dem die SPD noch in den 1950er und 1960er Jahren schöpfen konnte, hat sich beträchtlich reduziert. Der Anteil dieser Gruppen an der SPD-Wählerschaft ist zwischen 1953 und heute von über $60 \%$ auf deutlich unter $30 \%$ gesunken (ebd.). Dagegen hat die Bedeutung der Angestellten auf allen Ebenen zugenommen. Sie sind es auch, die eher wählen gehen, die am ehesten durch die Bilder von der „neuen Mitte“ angezogen wurden und zugleich auch am ehesten bereit sind, die „Seiten zu wechseln“.

\section{ENTWICKLUNG DER SPD-MITGLIEDSCHAFT}

Für die SPD ist die Stimmenmaximierung auf dem Wählermarkt das dominante Ziel. Zugleich wird die eigene Partei und deren Mitgliederentwicklung als wichtig erachtet, denn im Gegensatz zur CDU war die SPD schon unmittelbar nach dem Krieg eine ausgeprägte Mitgliederpartei, die mit über 700.000 Mitgliedern bereits 1946 über eine profunde Massenbasis verfügte. Die niedrigste Mitgliederzahl verzeichnete die SPD bislang im Jahr 1954: Sie rutschte nach dem katastrophalen Bundestagswahlergebnis des Jahres 1953 auf 585.479 Mitglieder ab. Ihren Mitgliederhöchststand erreichte sie 1976 mit einer Gesamtzahl von 1.022.191. Zwischen 1967 und 1976 wuchs die Mitgliederzahl um etwa 300.000 an. Seither gab es mit Ausnahme des Eini- 
gungsjahres 1990 keinen Mitgliederzuwachs mehr. Zwischen 1976 und 2007 ist die Mitgliederzahl der SPD um etwa $47 \%(=476.968)$ gesunken (Niedermeyer 2007).

Während die Sozialdemokratie den sozialstrukturellen Wandel der Bundesrepublik in ihrer Mitgliederstruktur näherungsweise nachvollziehen konnte, sind die Gewerkschaften bislang nicht in der Lage gewesen, diese Entwicklung in der eigenen Mitgliedschaft abzubilden (Tabelle 1). Letzteres zeigt sich besonders zugespitzt im Verhältnis zwischen Arbeitern und Angestellten. Zwar ist auch in den DGB-Gewerkschaften der Angestelltenanteil gewachsen, aber nicht parallel zum realen Anstieg der Angestellten unter den Beschäftigten. Einem Arbeiteranteil von $53 \%$ in den Gewerkschaften stehen weniger als $15 \%$ in der SPD gegenüber.

\section{Optionen im Verhältnis zwischen SPD und Gewerkschaften}

Vor dem Hintergrund der skizzierten sozialstrukturellen Veränderungen in der Wähler- und Mitgliederstruktur von SPD und Gewerkschaften werden nun einige systematische Überlegungen angestellt. Das Verhältnis zwischen Gewerkschaften und SPD kann unter den Bedingungen sozialdemokratischer Regierungsbeteiligung drei unterschiedliche Formen annehmen:

(1) Anpassung bzw. Unterordnung der Gewerkschaften: Diese Option besagt, dass die Gewerkschaften auf die Bildung eigener offensiver Akzente verzichten. Dies heißt nicht automatisch, dass sie sich unkritisch verhalten. Sie kritisieren die Regierungspolitik in einzelnen Punkten und praktizieren zugleich auch einen situativen Lobbyismus. Sie versuchen jedoch nicht, für ihre besonderen Interessen zu mobilisieren, was die Regierungsstabilität gefährden könnte. Diese Variante der Unterordnung kann sich in der Bandbreite vom aktiven Transmissionsriemen in die Gesellschaft bis hin zum stillschweigenden Mitspieler bewegen.

(2) Konfliktpartnerschaft: Die Gewerkschaften pendeln zwischen Kooperation und Konflikt. Einmischung und aktive Beteiligung der Gewerkschaften bis hin zur

Tabelle 1: Anteile der Arbeiter, Angestellten und Beamten in Gesellschaft, SPD und DGB - in \% -

\begin{tabular}{|c|c|c|c|c|c|c|c|}
\hline & & 1950 & 1970 & 1980 & 1990 & 2000 & $2004 *$ \\
\hline \multirow[t]{3}{*}{ Arbeiter } & Gesellschaft & 48,8 & 47,4 & 42,3 & 37,4 & 33,4 & 29,9 \\
\hline & SPD & 45,0 & 34,5 & 27,4 & 26,0 & 19,3 & 12,1 \\
\hline & DGB & 83,2 & 75,8 & 68,2 & 66,6 & 60,2 & 52,8 \\
\hline \multirow[t]{3}{*}{ Angestellte } & Gesellschaft & 16,5 & 29,6 & 37,2 & 43,3 & 48,5 & 51,2 \\
\hline & SPD & 17,0 & 20,6 & 23,4 & 26,6 & 27,8 & 23,9 \\
\hline & DGB & 10,5 & 14,7 & 21,0 & 23,3 & 28,6 & 30,8 \\
\hline \multirow[t]{3}{*}{ Beamte } & Gesellschaft & 4,1 & 5,5 & 8,4 & 8,5 & 6,8 & 6,6 \\
\hline & SPD & 5,0 & 9,9 & 9,4 & 10,8 & 10,7 & 9,1 \\
\hline & DGB & 6,3 & 9,5 & 10,8 & 10,1 & 7,2 & 7,5 \\
\hline \multirow[t]{3}{*}{ Andere** } & Gesellschaft & 30,6 & 17,5 & 12,1 & 10,8 & 11,3 & 12,3 \\
\hline & SPD & 33,0 & 35,0 & 39,8 & 36,6 & 42,2 & 54,9 \\
\hline & DGB & 0,0 & 0,0 & 0,0 & 0,0 & 4,0 & 8,9 \\
\hline
\end{tabular}

Personalunion wird von beiden Seiten für wichtig gehalten. Dabei werden seitens der Gewerkschaften auch durchaus aktiv eigene Konzepte öffentlich kommuniziert und beworben. Entscheidend ist jedoch, dass eine grundsätzliche Opposition zur SPD und eine Absage an deren Regierungsfähigkeit nicht angestrebt werden, solange erkennbar ist, dass es sich um eine Politik handelt, die im Rahmen bestehender Möglichkeiten gewerkschaftliche Interessen berücksichtigt.

(3) Autonome Gewerkschaftspolitik: Diese Option geht davon aus, dass die beiden großen Parteien - sowohl als „große Koalition" in der Regierung wie auch getrennt in Regierung und Opposition - auf der inhaltlichen Ebene gewissermaßen einen monolithischen Block bilden. Angesichts unvereinbarer Interessen und Strategien sowie sich derart „,neoliberal“ verhaltender sozialdemokratischer Parteien gehen die Gewerkschaften ihren eigenen Weg bzw. versuchen mittels gesellschaftlicher Mobilisierung von der SPD aufgekündigte Positionen erneut parteipolitisch zu verankern. Deshalb konzentrieren sich die Gewerkschaften in diesem Modell auf eine Mobilisierung der Gesellschaft und der Mitglieder durch Demonstrationen bis hin zur Gründung bzw. Unterstützung einer „Linkspartei", um ihre Interessen auch jenseits der SPD artikulieren bzw. parteipolitisch verankern zu können.

Bei diesen Optionen handelt sich selbstverständlich um Idealtypen. In der Realität ist das Verhältnis der Gewerkschaften zur SPD zwischen diesen Idealtypen angesiedelt, mit einer leichten Tendenz zur Konfliktpartnerschaft, wobei unterschiedliche Aus- prägungen bei den einzelnen DGB-Gewerkschaften wie auch für einzelne historische Phasen festzustellen sind.

\section{$(2$ \\ Verbindungslinien zwischen SPD und Gewerkschaften}

Es gibt zwei wesentliche Dimensionen, die sich im Verhältnis zwischen Gewerkschaften und SPD verändert haben: Erstens hat sich die sozialstrukturelle Basis von Gewerkschaften und Sozialdemokratie in den letzten Jahren auseinander entwickelt. Und zweitens haben sich die jeweiligen Arenen, in denen die beiden Akteure agieren (Parlamentarismus und Tarifautonomie) deutlich gewandelt. Schnittmengen sind schwieriger herzustellen. Beides müssen aber keine Gründe für einen grundlegenden politischen Entfremdungsprozess sein. Denn die realen Interessenunterschiede können durch übereinstimmende Deutungen der Situationen, Handlungsinstrumente, durch Strategien des (Aus)Tauschs und der Beteiligung zumindest relativiert werden. Von besonderer Bedeutung ist in diesem $\mathrm{Zu}$ sammenhang eine flexible Kooperation der jeweiligen Eliten auf der Basis gemeinsamer Leitbilder. Und tatsächlich gibt es vielfältige inhaltliche und programmatische Gemeinsamkeiten (Schabedoth 2008), die nicht alleine mit einer ähnlichen Herkunft und einer gemeinsamen Verantwortung in der Gegenwart zu tun haben.

\subsection{PROGRAMMATIK}

Die Struktur der Einheitsgewerkschaft verbietet eine direkte Option zugunsten der 
SPD im Wahlkampf. Gleichwohl besteht auf der Ebene der Grundwerte, der Ziele und Instrumente eine traditionell enge Beziehung, die wir durchaus als privilegierte Partnerschaft bezeichnen können. Im Gegensatz zur CDU/CSU spricht die SPD den Gewerkschaften in ihren Programmen eine herausragende Rolle als positiver Faktor gesellschaftlicher Integration und Gestaltung zu, was im Godesberger (1959), im Berliner (1989) aber auch im Hamburger Programm (2007) deutlich herausgestellt wird. Besonders verbindend ist bisher der weitgehende Konsens zwischen SPD und DGB über die Leistungen und die Leistungsfähigkeit des deutschen Modells von Sozialstaat und industriellen Beziehungen, vor allem die Anerkennung von Tarifautonomie und Mitbestimmung als fester Bestandteil der SPD-Regierungspolitik. Alles, was diesen Konsens erschüttert oder aufzukündigen scheint, wird umso sensibler wahrgenommen, so z. B. das "SchröderBlair-Papier 1999" sowie eine sozialdemokratische Regierungspolitik, die auf Privatisierung, Eigeninitiative und eine Reduktion des Staates im Sinne einer neu akzentuierten Aktivierungsstrategie setzte, nicht zuletzt auch durch die „Rente ab 67“.

Die Agenda 2010 wird seitens der Mehrheit der Gewerkschafter als eine angebotsorientierte Politik, aber auch als eine ideologische Entkopplung von den gemeinsamen Wurzeln verstanden, womit zudem die soziale Symmetrie gefährdet werde. Während die Gewerkschaften in ihren Diskursen mit der Sozialdemokratie die Bedeutung der Verteilungsgerechtigkeit herausstellen, besteht die Mehrheit der SPD-Führung darauf, dass dieses Gerechtigkeitskonzept relativiert werden müsse und Teilhabe-, Chancen- wie auch Generationengerechtigkeit ebenfalls eine wichtige Rolle spielen sollten. Vor diesem Hintergrund wuchs bei einigen Funktionären der Gewerkschaften die Bereitschaft, sich nicht nur von der sozialdemokratischen Regierungspraxis zu distanzieren, sondern auch eine eigene Partei links von der SPD aufzubauen. Auch wenn deren Zustandekommen letztlich von einer Vielzahl von Zufällen getragen war, bleibt festzuhalten, dass die handelnden Akteure das ,window of opportunity" nutzten, um eine kommunikative Plattform zu schaffen, die eng an den programmatischen Positionen der beiden großen Gewerkschaften IG Metall und ver.di orientiert ist. Erst der SPD-Vorsitzende Beck konnte, unterstützt durch Ver- änderungen an der „Agenda 2010“ und flankiert durch das neue Hamburger SPDGrundsatzprogramm (2007), die SPD-Politik auch emotional wieder in die Nähe gewerkschaftlicher Befindlichkeiten rücken, ohne damit die neue sozialdemokratische Politik aufzugeben.

\subsection{PERSONELLE VERFLECHTUNGEN}

Gerade angesichts einer auseinanderfallenden sozialstrukturellen Basis sind die SPDund Gewerkschaftseliten besonders gefordert, inhaltliche Schnittmengen und gemeinsame Projekte zu definieren. Gute Voraussetzungen dafür sind allemal vorhanden, schon allein aufgrund persönlicher und personeller Verflechtungen. Gewerkschafter wählen mehrheitlich SPD; ein Teil von ihnen unterstützt sie nach wie vor aktiv. Und SPD-Mitglieder sind auch Gewerkschafter: Etwa ein Drittel aller SPDMitglieder ist gewerkschaftlich organisiert. Quantitativ betrachtet kommt die Majorität der sozialdemokratischen Gewerkschaftsmitglieder aus den Verbänden des öffentlichen Dienstes. So liegt etwa der ver.di- und der GEW-Anteil unter den gewerkschaftlich organisierten Sozialdemokraten deutlich über ihrem DGB-Anteil, während die IG Metall in der SPD deutlich unterrepräsentiert ist. Doppelmitgliedschaften, also SPD-Parlamentarier und Gewerkschaftsmitglied zu sein, führen - gestützt durch klare Rollendefinitionen nicht zu tief gehenden Konflikten. Im Gegenteil: Gewerkschaften benötigen einen Zugang zum politischen System. Ebenso kann es für eine sozialdemokratische Partei in Regierung und Parlament, die Konflikte mit der Arbeitnehmerschaft und ihren Gewerkschaften nicht bewusst in Kauf nehmen will, hilfreich sein, eine Rückbindung an die gewerkschaftliche Funktionselite zu haben. Die Voraussetzungen dafür sind nach wie vor gegeben: Mit Ausnahme des ver.di Vorsitzenden, der das Parteibuch der Grünen besitzt, sind alle DGB-Vorsitzenden zugleich auch SPD-Mitglieder.

Mit dem 1968 unter Willy Brandt gegründeten Gewerkschaftsrat besteht auch ein Gremium des regelmäßigen Austauschs zwischen Partei- und Gewerkschaftsspitzen, das insbesondere in Phasen des Konflikts den Charakter einer Clearingstelle annehmen kann. Und da alle Gewerkschaftsvorsitzenden mit sozialdemokratischem Parteibuch an diesen Gesprächen teilnehmen, kann diese Einrichtung auch dazu beitragen, die gewerkschaftlichen Positionen einander anzunähern. Auch die jahrzehntelange Gepflogenheit, führende Gewerkschafter als Minister in Regierungen unter SPD-Führung einzubinden (das Amt des Arbeitsministers) ist eine Form der Kontaktpolitik. Eine Zäsur bezüglich dieser Tradition gab es erstmals 2002 - bis heute.

Inwieweit die gewerkschaftlich organisierten Bundestagsabgeordneten ein Garant für einen intensiven Austausch zwischen SPD und Gewerkschaften sind, ist schwierig zu beantworten. Denn aus der Sicht der Parlamentarier kann die Mitgliedschaft in einer Gewerkschaft sowohl normative als auch instrumentelle Gründe haben. Weder das eine noch das andere sagt allerdings etwas über die Bereitschaft und Fähigkeit aus, gewerkschaftliche Interessen im politischen Raum zu vertreten. Gleichwohl hat der hohe Anteil von Gewerkschaftsmitgliedern bei sozialdemokratischen Bundestagsabgeordneten seitens der Unternehmerverbände immer wieder zu dem Vorwurf geführt, es handele sich bei der SPD-Bundstagsfraktion um eine "Gewerkschafts-Fraktion“ (Schmollinger 1973, S. 229). Tatsächlich aber spielen die gewerkschaftlich organisierten Bundestagsabgeordneten weder als Adressat entsprechender Zielgruppenarbeit eine wirklich wichtige Rolle noch sind Entscheidungen bekannt, bei denen diese Bundestagsabgeordneten im Konfliktfall und als Block Interessen der Gewerkschaften gegen die Optionen der Fraktions- und Parteispitze verfolgt hätten.

Und ohnehin: Seit 1990 ist bei allen Bundestagsfraktionen mit Ausnahme der PDS ein deutlicher Rückgang hinsichtlich des gewerkschaftlichen Organisationsgrades feststellbar. Die jüngere Generation der Abgeordneten setzt viel seltener als ihre Vorgänger auf das Prinzip überlappender Mitgliedschaften. Mit der Bundestagswahl 1990 sank der gewerkschaftliche Organisationsgrad in der SPD-Fraktion von über $90 \%$ auf rund $74 \%$, der der CDU/CSUFraktion von fast $20 \%$ auf 7,5\%. Diese Entwicklung hielt auch bei der Bundestagswahl 2005 an: Der Anteil der gewerkschaftlich organisierten Parlamentarier in der SPD-Fraktion ging weiter auf mittlerweile rund $59 \%$ zurück. Bei der CDU/CSU liegt er gegenwärtig bei unter $1 \%$. Aber nicht nur die Gewerkschaftsmitgliedschaft der Parlamentarier ist rückläufig. Auch die emotionale Bindung zu den Gewerkschaf- 
ten lockert sich. Vor allem bei den jüngeren Abgeordneten gibt es aufgrund fehlender Erfahrungen, anderer Herkunft und Sozialisation ein $\mathrm{Maß}$ an Entfremdung gegenüber den Gewerkschaften wie nie zuvor in der Geschichte des bundesdeutschen Parlamentarismus.

\section{Fazit und Ausblick}

Die Beziehungen zwischen SPD und Gewerkschaften haben sich deutlich verändert. Sie sind programmatisch und personell weniger eng, komplizierter und emotional schwächer geworden. Die Distanz, die sich hier zeigt, erklärt sich nicht allein aus zyklisch-konjunkturellen Schwankungen, wie sie immer schon bestanden haben, insbesondere in Abhängigkeit davon, ob sich die SPD in der Opposition oder in Regierungsverantwortung befand. Hinzu kommen offensichtliche Prozesse ideologischer Entkopplung, insbesondere während der Kanzlerschaft von Gerhard Schröder. Die Hauptursache aber ist, dass die Mitglieds- bzw. Wählerschaften von Gewerkschaften und SPD hinsichtlich ihrer sozialstrukturellen Zusammensetzung deutlich auseinanderdriften.

Umso wichtiger wird der kommunikative, diskursive Austausch als ein wichtiges Verbindungsglied zwischen SPD und Gewerkschaften. Denn ob die Beziehungen zwischen SPD und Gewerkschaften besser oder schlechter funktionieren, hängt nicht unwesentlich davon ab, wie angesichts vorhandener struktureller und politischer Divergenzen die Spitzen „miteinander können“, ob sie zu gemeinsamen Lagedeutungen, gegebenenfalls sogar Handlungsorientierungen gelangen. Die Chancen dafür stehen nicht schlecht, solange es wie bei den heutigen Eliten auf Seiten der Gewerkschaften wie auch der SPD noch einen breiten Korridor gemeinsamer Überzeugungen gibt. Doch dies darf nicht darüber hinwegtäuschen: Hinter den Spitzen, also auf den mittleren Funktionärsrängen, besteht mittlerweile vielfach ein Nichtverhältnis. Von Grund auf belastbare Strukturen sind am ehesten noch in industriellen Zentren mit sozialdemokratischer Dominanz vorhanden.

Festzuhalten bleibt aber auch: Selbst nach dem Parlamentseinzug der Linkspartei, die für „old labour" steht, sind die Be- ziehungen zwischen Gewerkschaften und Sozialdemokratie immer noch intensiver und umfangreicher als zu jeder anderen Partei. So haben im Jahre 2005 etwa 47 \% der Gewerkschaftsmitglieder SPD gewählt, $24 \%$ die CDU und $13 \%$ die Linkspartei (ARD/Infratest dimap Wahlbefragung 2005). Auf zwei wesentlichen Ebenen Mitglieder- und Wählerebene - gibt es vielfältige Überschneidungen, wenn auch mit abnehmender Tendenz. Die deutlichen Mitglieder- und Wählerverluste der SPD sind Folge ihrer Verluste in ihrem Kernmilieu, bei den Arbeitnehmern, ohne dass sie im gleichen Umfang andere Gruppen gewinnen oder stärker an sich binden konnten. Aber während hinsichtlich der Wahlentscheidung noch keine dramatische Erosion oder gar Entkopplung zwischen SPD und gewerkschaftlich organisierter Wählerschaft festzustellen ist, hat sich das inhaltliche Verhältnis geändert. Die SPD zu wählen, ist bei dieser Wählerschicht zunehmend weniger ein Zeichen von emotionaler, organischer Zuneigung, sondern eher Ausdruck instrumenteller, rationaler Präferenz.

Die Gewerkschaften haben ihren Einfluss im politischen System bislang ihrer Fähigkeit zuzuschreiben, einen wesentlichen Teil der Arbeitnehmerschaft repräsentieren zu können. Gefährdet werden könnte dies, wenn die bis heute unzureichenden Erfolge in Zukunftsbranchen, bei den neuen Berufen, in den kleinen Betrieben - und nicht zu vernachlässigen bei Frauen sowie Jugendlichen - andauern. Der Einfluss kleiner Spartengewerkschaften ist zwar bislang noch vergleichsweise gering; es könnte aber sein, dass die von diesen kleinen, homogenen Organisationen praktizierte Offensivstrategie Schule macht und damit die großen Gewerkschaften zusätzlich unter Druck setzt. In diesem Sinne kann auch die abnehmende Verankerung der Gewerkschaften bei relevanten Teilen der abhängig Beschäftigten, vor allem bei den Höherqualifizierten, dazu führen, dass ihnen weitere Einflussmöglichkeiten versperrt bleiben. Welche Gestaltungsmacht die Gewerkschaften zukünftig haben werden, wird schließlich auch davon abhängen, ob sie mit den Entwicklungen des Arbeitsmarktes auf Augenhöhe bleiben. Gelingt ihnen wieder eine neue Aufgeschlossenheit, dann könnte auch ein Negativszenario zur Zukunft von Gewerkschaften und SPD verhindert werden: Denn ob in Schwäche vereint oder getrennt - beides wäre keine verlockende Perspektive.
Für die SPD bedeutet die dauerhafte Parlamentarisierung der Linkspartei, dass sie diese als zusätzliche Option in ihre Machtpolitik einbeziehen muss, um die eigene Regierungsfähigkeit in einem Fünfoder Sechsparteiensystem zu ermöglichen. Dies heißt jedoch keinesfalls, dass sie damit strukturell nach links rücken müsste; ihr könnte-machtpolitisch gesehen - auch bereits der hinzugewonnene Flexibilitätsspielraum genügen. Das heißt: Wenn es den Gewerkschaften nicht gelingen würde, sich in der Dienstleistungs- und Wissensökonomie (und damit in wichtigen gesellschaftlichen Zentren bzw. Mitglieder- und Wählerschichten) stärker zu verankern, dann müsste auch die SPD auf gewerkschaftliche Interessen zunehmend weniger Rücksicht nehmen. Die SPD könnte sich dann in sozialstaatlichen Fragen problemloser mittig oder sogar in Richtung liberaler Positionen bewegen. Zugleich würde die SPD infolge einer solchen Konstellation in nahezu jeder Situation, die mittelfristig absehbar ist, eine entscheidende Rolle für die Mehrheitsverhältnisse im Parlament spielen, wenn sie klug und flexibel agiert. Und in den Gewerkschaften könnte als Reaktion auf die flexible Pendelpolitik der SPD sogar die Bewegung in Richtung Linkspartei einen stärkeren Schub erhalten, den es in pointierter Form zurzeit nicht gibt.

Von einer neuen Flexibilität der SPD im Fünfparteiensystem können also durchaus Gefahren für die Gewerkschaften ausgehen. Die theoretisch denkbare Option der Gewerkschaften, die privilegierte Partnerschaft mit der SPD gegen eine mit der Linkspartei auszutauschen, dürfte real kein begehbarer Pfad sein: Hiermit zerfiele nicht nur ihr Status als Einheitsgewerkschaft; ungleich erschwert wären auch die Voraussetzungen, Veränderungen am Arbeitsmarkt und Verschiebungen in den Beschäftigtenstrukturen organisationspolitisch abzubilden. Wollen die Gewerkschaften die im Gange befindliche Einbettungskrise nicht verschärfen, dann wären sie gut beraten, sich in ihrer Politik an den Veränderungen des Arbeitsmarktes zu orientieren, ohne ihre kritische und mobilisierungsfähige Unabhängigkeit gegenüber dem politischen System zu gefährden. Durch ihre derzeit beobachtbare Politik der inneren Konsolidierung scheinen sie auf einem guten Weg zu sein, sich gewissermaßen nachholend mit den veränderten Wirklichkeiten zu versöhnen, um so die Voraussetzungen für eine neue Einflusspolitik zu schaffen. 
Armingeon, K. (1988): Die Entwicklung der westdeutschen Gewerkschaften 1950-1985, Frankfurt a. M./New York

Müller-Jentsch, W./Ittermann, P. (2000): Industrielle Beziehungen. Daten, Zeitreihen, Trends 1950-1999, Frankfurt a. M.

Piazza, J. (2001): De-linking Labor. Labor Unions and Social Democratic Parties under Globalization. in: Party Politics 4, S.413-435

Pirker, T. (1960): Die blinde Macht. Die Gewerkschaftsbewegung in Westdeutschland 1945-1955, 2 Bde., München

Niedermeyer, O. (2007): Parteimitgliedschaften im Jahre 2006, in: Zeitschrift für Parlamentsfragen (ZParl) 2, S. 368-375

Roth, D. (2003): Das rot-grüne Projekt an der Wahlurne, in: Egle, C./ Ostheim, T./Zohlnhöfer, R. (Hrsg.): Das rot-grüne Projekt, Opladen, S. 29-52

Schabedoth, H. J. (2008): Blick auf die Grundsatzprogramme der Volksparteien - Eine gewerkschaftliche Position, in: WSI-Mitteilungen 2,

S. $107-113$

Schmollinger, H. W. (1973): Gewerkschafter in der SPD - Eine Fallstudie, in: Jürgen, D./Ebbighausen R. (Hrsg.): Parteiensystem in der Legitimationskrise, Opladen, S.229-274
Schneider, M. (1994): Darstellung zur Geschichte des Bündnisses von SPD und Gewerkschaften, in: Langkau, J./Matthöfer, H./Schneider, M. (Hrsg.): SPD und Gewerkschaften - Zur Geschichte eines Bündnisses (Band 1), Bonn, S. 12-74

Silvia, S. J. (1992): The Forward Retreat - Labor and Social Democracy in Germany 1982-1992, in: International Journal of Political Economy 4, S. $36-52$

Wessels, B. (2000): Gruppenbindungen und Wahlverhalten - 50 Jahre Wahlen in der Bundesrepublik, in: Klein, M./Jagodzinski, W./Mochmann, E./Ohr, D. (Hrsg.): 50 Jahre empirische Wahlforschung in Deutschland, Wiesbaden, S. 129-158

Wessels, B. (2007): Organisierte Interessen und Rot-Grün - Temporäre Beziehungsschwäche oder zunehmende Entkopplung zwischen Verbänden und Parteien?, in: Egle, C./ZohInhöfer, R. (Hrsg.): Ende des rot-grünen Projektes. Eine Bilanz der Regierung Schröder 2002-2005, Wiesbaden, S. 151-167 